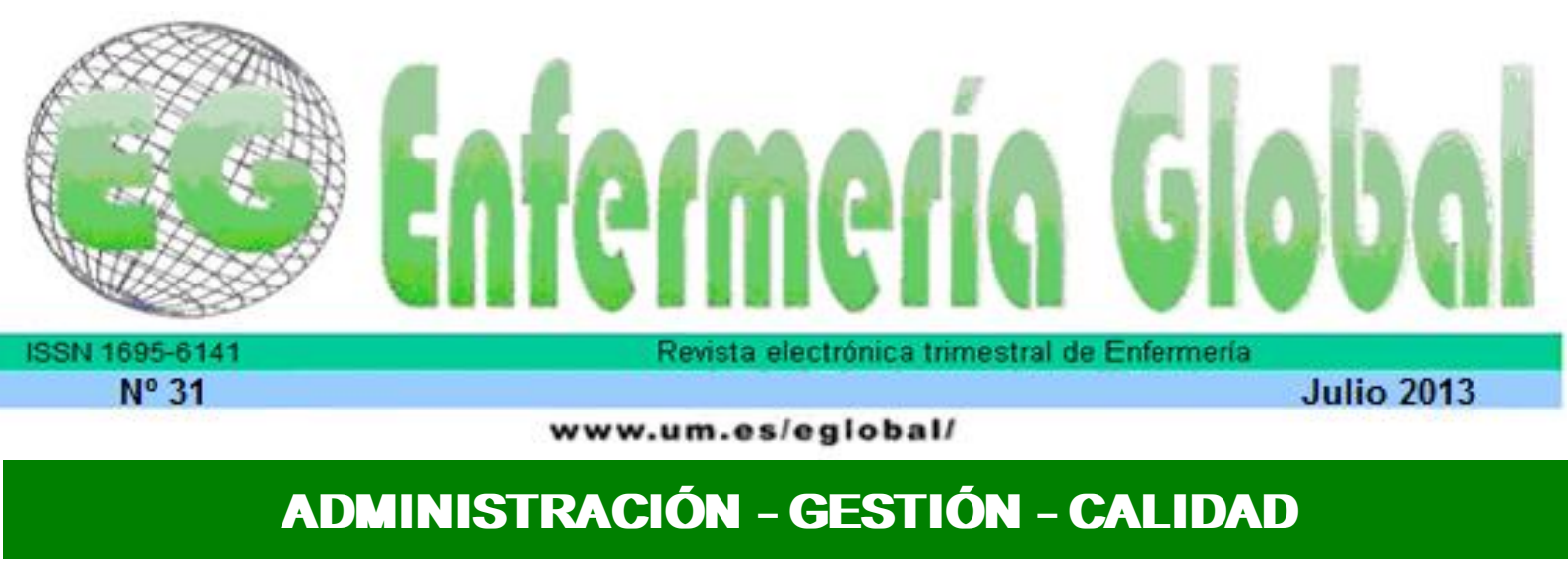

\title{
Programación del cambio de turno desde la óptica de los profesionales de enfermería
}

Mapeamento da passagem de plantão sob a ótica dos profissionais de enfermagem

Shift change mapping the perspective of nursing professionals

*Llapa Rodriguez, Eliane Ofelia ${ }^{* *}$ Oliveira, Cleiton da Silva ${ }^{* *}$ de França, Tâmara Raylane Santos ${ }^{* * *}$ de Andrade, Joseilze Santos *Pontes de Aguiar Campos, Maria ***Pinto da Silva, Flávia Janólio Costacurta

\begin{abstract}
*Profesora doctora del Departamento de Enfermería. E-mail: elianaofelia@gmail.com **Estudiante del Curso de Enfermería. ***Profesora Máster del Departamento de Enfermería. Universidad Federal de Sergipe - UFS. Brasil.

Palabras clave: asistencia integral a la salud; administración hospitalaria; comunicación; enfermería; planeamiento
\end{abstract}

Palabras chave: assistência integral à saúde; administração hospitalar; comunicação; enfermagem; planejamento.

Keywords: Integral health attendance; hospital management; communication; nursing; planning

\section{RESUMEN}

Objetivo: Analizar la influencia de los factores internos y externos (Fortalezas, Oportunidades, Debilidades y Amenazas) durante el proceso de cambio de turno, según la óptica de profesionales de enfermería del Hospital Universitario de Sergipe, Brasil.

Método: Investigación de tipo descriptiva-exploratoria con enfoque cuantitativo utilizando el planeamiento estratégico como fundamento teórico, así como la construcción de la Matriz de Fortalezas, Oportunidades, Debilidades y Amenazas (FODA). Los datos fueron recolectados a través de cuestionario previamente elaborado.

Resultados: Tipos de pasaje de turno encontrados: entre profesionales de la misma categoría, a través del libro, en la unidad del paciente. Fortalezas: duración máxima de 15 a 20 minutos, objetividad en las informaciones y cooperación del equipo. Debilidades: funcionarios apresurados para salir, impuntualidad para inicio y atraso de los colegas. Oportunidades: relación efectiva con otros profesionales, la gerencia hospitalaria y los nuevos modelos de gestión implantados en la institución. Amenazas: llamadas telefónicas durante el cambio de turno, exceso de unidades bajo responsabilidad de los profesionales. Se elaboró también la matriz estratégica FODA.

Conclusiones: Posterior al estudio sistemático de los factores internos e externos fue posible proponer alternativas capaces de contribuir en la gestión del cuidado. 


\title{
RESUMO
}

Objetivo: Analisar a influência dos fatores internos e externos (Fortalezas, Oportunidades, Fraquezas e Ameaças) no processo de passagem de plantão, segundo a ótica dos profissionais de enfermagem do Hospital Universitário de Sergipe, Brasil.

\begin{abstract}
Método: Pesquisa do tipo descritivo-exploratório com abordagem quantitativa utilizando o planejamento estratégico como referencial teórico para a construção da Matriz de Fortalezas, Oportunidades, Fraquezas e Ameaças (FOFA). Os dados foram coletados utilizando questionário previamente elaborado.

Resultados: Foram encontrados como tipos de passagem de plantão: enfermeiro passa para enfermeiro e auxiliar para auxiliar, através do livro de plantão, à beira do leito; como fortalezas: duração máxima de 15 a 20 minutos para a passagem de plantão, objetividade nas informações e cooperação da equipe; como fraquezas: funcionários apressados para sair, impontualidade para o início e atraso dos colegas; como oportunidades: relacionamento com outros profissionais, a administração hospitalar e novos modelos de gestão implantados na instituição; e, como ameaças: chamadas telefônicas durante a passagem de plantão, excesso de unidades sob responsabilidade de um mesmo enfermeiro, a administração hospitalar. Apresentou-se também a construção da matriz estratégica FOFA.
\end{abstract}

Conclusões: após estudo sistemático dos fatores internos e externos, foi possível gerar alternativas estratégicas capazes de contribuir para a melhoria da gestão do cuidado.

\section{ABSTRACT}

Objective: To analyze the influence of the internal and external factors (Strengths, Opportunities, Weakness and Threatens) during the process of shift change, according to the view of nursing professionals at the University Hospital of Segirpe, Brasil.

Method: Descriptive-exploratory research, with a quantitative approach using the strategy plan, as a theoretical basis, as much as the building of the Strengths, Opportunities, Weakness and Threatens matrix (SOWTM). Data were collected through a previous elaborated questionnaire.

Results: Types of shift change that were found: among professionals of the same category, by means of the book, in the unit of the patient. Strengths: maximum duration from 15 to 20 minutes, objective information and team co-operation. Weakness: civil servants in a hurry because they wanted to go home, lack of punctuality to start and delay of the colleagues. Opportunities: effective relations with other professionals, the hospital management and the new models of administration settled in the institution. Threatens: Phone calls during the shift change and too many units under the professionals' responsibility. The strategy matrix (SOWTM) was also elaborated.

Conclusions: After the systematic study of the internal and external factors, it was possible to propose alternatives that could contribute to the care management.

\section{INTRODUCCIÓN}

El personal de enfermería se encarga de la atención integral al cliente y de su continuidad. Con la intención de garantizar la prestación de esta asistencia, el enfermero utiliza como estrategia el procedimiento de cambio de turno, por ser esta una actividad comunicativa y de rutina, esencial e inherente a su trabajo diario, el cual le permite organizar y planificar las intervenciones de enfermería a ser realizadas.

La comunicación es un proceso fundamental que permite la comprensión e intercambio de información. En enfermería esta herramienta es muy apreciada entre los profesionales, ya que favorece la gestión del cuidado asegurando su eficiencia y eficacia ${ }^{(1)}$. Concomitantemente, el cambio de turno utiliza la comunicación como una forma de asegurar el flujo rápido de información relativa al cotidiano de trabajo del 
enfermero, para de esta forma transmitir, recibir y delegar tareas entre cada equipo, actividad que implicará una activa interacción entre los profesionales ${ }^{(2)}$. Así mismo, este procedimiento garantiza la identificación de problemas y necesidades para su posterior análisis situacional, lo cual permitirá una constante reorientación en la planificación del cuidado de enfermería ${ }^{(1)}$.

Entre los diferentes tipos de cambio de turno observados tenemos los cambios por tareas, por grupos y subgrupos, por informe verbal/escrito, la entrega de turno en la unidad del paciente y aquella realizada de enfermera para enfermera y de auxiliar de enfermería para auxiliar de enfermería de forma separada ${ }^{(2,3)}$.

Durante el cambio de turno, los contenidos informados se refieren a situaciones asistenciales - administrativas que envuelven al paciente y al sector de internación. Las informaciones deben incluir: la identificación del paciente, diagnóstico médico, las complicaciones y los cambios en el estado clínico del paciente, cuidados de enfermería en general, las modificaciones en las prescripciones médicas, características de dolor y tratamiento, tipo, programación y preparación para exámenes, informaciones relacionadas con el balance hídrico; tipo y porcentaje de la dieta consumida, el estado de la incisión quirúrgica y las características de la herida, resultados de pruebas y exámenes, información sobre equipos y accesorios utilizados por el paciente, por citar los principales ${ }^{(4.5)}$.

Sin embargo, existen factores que afectan ael cambio de turno, se citan como positivos: la organización previa, la concentración dada a este procedimiento, la puntualidad al inicio y al final del procedimiento, el entorno tranquilo y amplio ${ }^{(2)}$. Como aspectos negativos se mencionan: el poco valor dado al cambio de turno, la falta de comunicación y la claridad en los registros, el excesivo número de pacientes en las unidades hospitalarias, los retrasos del personal de enfermería, la documentación insuficiente, la sobrecarga de trabajo en la supervisión, el constante flujo de otros profesional en la unidad, las llamadas telefónicas, y el tiempo insuficiente para la transmisión de información ${ }^{(3,6)}$.

En este contexto, para que el procedimiento de cambio de turno alcance el éxito gerencial, de forma que contribuya a una atención de calidad, es necesario el uso de algunas acciones que deben ser integradas al trabajo del equipo de enfermería, entre ellas la planificación, considerando que esta es una actividad realizada de forma predominante por el enfermero ${ }^{(7)}$.

La planificación es un proceso continuo que se puede definir como el arte de la toma de decisiones y la creación de planes para conducir un proceso de cambio. Su objetivo es proporcionar una gestión activa en sus relaciones con los clientes y con el entorno en el cual interactúa, con la intención de intervenir en la realidad y favorecer el alcance de las metas preestablecidas ${ }^{(8)}$.

Para la administración en general, la planificación se clasifica en tres tipos: estratégica, táctica y operativa, que corresponden, respectivamente, a los niveles jerárquicos, intermedios y operativos. Sin embargo, en la administración en salud la planificación se divide en normativa (o tradicional) y la Planificación Estratégica Situacional (PES) ${ }^{(8.9)}$. La planificación estratégica es un proceso que tiene como objetivo establecer metas y proponer estrategias para alcanzarlas, a partir de un cuidadoso análisis de su entorno interno y externo, proporcionando una mejor 
utilización de los recursos, lo que favorece que la organización se pueda preparar para cambios probables que podrían desestabilizarla ${ }^{(10)}$.

La planificación estratégica utiliza la matriz de Fortalezas, Oportunidades, Debilidades y Amenazas (FODA) para establecer la conexión entre los puntos fuertes y débiles de la organización. Las fortalezas y debilidades analizan el entorno interno, que se compone principalmente de los recursos humanos, físicos y organizacionales, mientras que las amenazas y oportunidades analizan el entorno externo, tomando como base las fuerzas político-legales, económicas, sociales y tecnológicas ${ }^{(11)}$.

En este sentido, la planificación estratégica será utilizada con el objetivo de programar los factores internos y externos y su influencia en el procedimiento de cambio de turno. A partir de esta situación será posible identificar los tipos de cambio de turno y analizar las fortalezas y debilidades con la finalidad de sugerir posibles intervenciones.

En este contexto, se tuvo como objetivo general analizar la influencia de factores internos y externos (Fortalezas, Oportunidades, Debilidades y Amenazas) en el procedimiento de cambio de turno, según la óptica del personal de enfermería de un hospital universitario y como objetivos específicos identificar los tipos de cambio de turno utilizados en la unidades estudiadas, programar los factores internos y externos que influyen en el proceso de cambio de turno y construir la matriz FODA resultante de la programación de los factores internos y externos que influyen en el cambio de turno, en el mencionado hospital.

\section{MATERIALES Y MÉTODOS}

Estudio descriptivo- exploratorio, con enfoque cuantitativo. Se utilizó la planificación estratégica como referencial teórico, el cual permitió la construcción del instrumento.

La investigación se realizó entre marzo y julio de 2011, con el personal de enfermería del Hospital Universitario de la Universidad Federal de Sergipe, Brasil, en las clínicas médicas I y II, clínica quirúrgica I, Unidad de Cuidados Intensivos (UCI) y en la clínica pediátrica, durante los tres turnos, de lunes a domingo. La población estaba compuesta por 104 profesionales, distribuidos de la siguiente manera: 14 técnicos / auxiliares de enfermería en la clínica médica I, 18 técnicos / auxiliares de enfermería en la clínica medicina II, 16 técnicos / auxiliares de enfermería en la clínica quirúrgica I, 16 técnicos / auxiliares enfermería en pediatría, 18 técnicos / auxiliares de enfermería en la UCI, y 22 enfermeros distribuidos en todas las unidades citadas.

Fueron seleccionados profesionales que trabajaron al menos seis meses en la atención directa al paciente, así como aquellos que se encontraron presentes en el servicio durante el período de recolección de datos. El proyecto fue presentado a la Comisión de Ética de la Universidad Federal de Sergipe (CEP / UFS), siendo aprobado según protocolo 0020.0.107.000-11. Siguiendo los preceptos de la Resolución 196/96 del Consejo Nacional de Salud (CNS), los sujetos fueron informados de los objetivos y propósitos de la investigación.

Para la recolección de datos se utilizó un cuestionario previamente elaborado conformado de dos partes, la primera relativa a los datos de identificación de los profesionales investigados, y la segunda relativa a los datos específicos de la investigación, tales como tipos de cambio de turno, sus ventajas y desventajas, así 
como su factores internos y externos que influyen en ese proceso. Posteriormente fueron elaboradas posibles estrategias de intervención a partir del análisis de los factores analizados y sus respectivos cruzamientos (Cuadro 1).

El análisis de datos se fundamentó en el método de planificación estratégica propuesto por Stoner y Freeman ${ }^{(12)}$. Para los autores, la planificación estratégica es el punto de partida en la gestión estratégica de las organizaciones, independientemente de su tamaño y tipo. Este tipo de planificación tiene como objetivo emprender acciones decisivas, así como proponer resultados para conducir actitudes proactivas en la gestión organizacional ${ }^{(13)}$. Este método puede ser condensado en cuatro pasos básicos: el establecimiento de objetivos, la definición de la situación actual, la determinación de las facilidades y barreras, y la preparación de un conjunto de medidas (estrategias) que facilitan el establecimiento de metas ${ }^{(12)}$. Utilizando este método fue posible programar los factores internos, los factores externos y su influencia en el procedimiento de cambio de turno. Destaca que la elección de la metodología fue adecuada para este trabajo debido a que la planificación presupone la adopción de filosofías, las cuales permiten que la institución pueda obtener una visión del futuro.



Fuente: Lip (1998) ${ }^{(22)}$.

\section{RESULTADOS Y DISCUSIÓN}

Por ser un estudio descriptivo, la primera parte de los resultados se presenta en tablas que muestran frecuencias y porcentajes, determinando así el perfil sociodemográfico de los profesionales de enfermería que trabajan en el hospital analizado. Los resultados mostraron que la mayoría de los profesionales pertenece a la categoría de técnicos / auxiliares de enfermería $(77,1 \%)$, seguido por la categoría de enfermeros $(22,9 \%)$. Del total de profesionales, la mayoría pertenecen al sexo femenino, con el $95,45 \%$ para los enfermeros y el $85,37 \%$ para los técnicos / auxiliares, predominó el estado civil casado, con 68,18\% para los enfermeros, y el $57,32 \%$ para los técnicos / auxiliares. Con relación al aspecto financiero familiar, la mayoría de los enfermeros y los técnicos / auxiliares comparten sus gastos con el cónyuge, con $54,55 \%$ y $46,34 \%$, respectivamente. 
Con respecto al nivel de escolaridad, el $67,07 \%$ de los técnicos / auxiliares tienen nivel medio, mientras que el 32,93\% tiene nivel universitario. Respecto al tiempo de formación, tanto los enfermeros como los técnicos / auxiliares tienen un tiempo mayor a seis años, con el $95,45 \%$ y el $97,56 \%$, respectivamente. La variable tiempo de trabajo fue superior a seis años, con el $77,27 \%$ para los enfermeros y el $76,83 \%$ para los técnicos / auxiliares. Cuando se les preguntó acerca de otros empleos, ambas categorías profesionales mencionaron poseer más de un empleo, con el 81,82\% para los enfermeros y el $76,83 \%$ para los técnicos / auxiliares. En cuanto a la variable ingreso mensual, ambas categorías tienen un ingreso de más de cuatro salarios mínimos, con $95,45 \%$ para los enfermeros y $80,49 \%$ los técnicos / auxiliares.

Con relación a las modalidades de cambio de turno, los profesionales mencionaron predominio de los siguientes tipos: enfermero pasa el turno para enfermero y auxiliar pasa para auxiliar (por separado), el cambio de turno a través del libro (informe) y el enfermero pasa para el equipo de enfermería (Tabla 1). En consonancia, la literatura menciona la existencia de varios tipos de cambio de turno ${ }^{(2,3,14,15,16)}$, incluyendo los mostrados en los resultados de este estudio, los cuales tienen como finalidad crear condiciones para garantizar la continuidad y la calidad de la atención durante la jornada de trabajo. Basado en estas respuestas, se observó que los cambios de turno adoptados variaron de una unidad a otra, dependiendo de la dinámica de trabajo, como por ejemplo el cambio de turno en la unidad del paciente, que se lleva a cabo sólo en la $\mathrm{UCl}$, tal vez por tener un número reducido de los pacientes.

Tabla 1. Tipos de cambio de turno reportado por los profesionales de enfermería de un hospital universitario. Sergipe, Brasil, 2012.

\begin{tabular}{|c|c|c|c|c|}
\hline Tipos de cambio de turno & $\begin{array}{l}\text { Frecuencia } \\
\text { Enfermeros }\end{array}$ & $\%$ & $\begin{array}{c}\text { Frecuencia } \\
\text { Tec/Aux }\end{array}$ & $\%$ \\
\hline $\begin{array}{l}\text { Enfermero pasa para enfermero y } \\
\text { auxiliar pasa para auxiliar (por } \\
\text { separado) }\end{array}$ & 22 & 37,2 & 78 & 46,99 \\
\hline $\begin{array}{l}\text { Cambio de turno a través del libro } \\
\text { (informe) }\end{array}$ & 17 & 28,8 & 31 & 18,67 \\
\hline $\begin{array}{l}\text { Enfermero pasa el turno al equipo de } \\
\text { enfermería }\end{array}$ & 14 & 23,7 & 22 & 13,25 \\
\hline $\begin{array}{l}\text { Cambio de turno junto a la unidad del } \\
\text { paciente }\end{array}$ & 5 & 8,47 & 25 & 15,06 \\
\hline Cambio de turno en grupos & 1 & 1,69 & 6 & 3,61 \\
\hline Cambio de turno por subgrupos & 0 & 0,00 & 3 & 1,81 \\
\hline Otro tipo de cambio de turno & 0 & 0,00 & 1 & 0,60 \\
\hline Total & 59 & 100 & 166 & 100 \\
\hline
\end{tabular}

Fuente: Datos de la investigación

La segunda parte de los datos permitió el análisis de los factores internos y externos, usando como método la planificación estratégica. En este sentido, cuando se analizaron los factores internos (fortalezas) en relación con el cambio de turno, según la percepción de los enfermeros, se encontraron como principales fortalezas: información clara, un tiempo máximo de 15 a 20 minutos para el cambio de turno, la objetividad de la información, ambiente tranquilo, el intercambio ágil de información, la cooperación y el compromiso del equipo de enfermería. Por otro lado, los técnicos / 
auxiliares de enfermería mencionaron la cooperación en equipo, duración máxima de 15 a 20 minutos para el cambio de turno, el compromiso del equipo, la objetividad de la información, la claridad de la información, la agilidad para intercambiar información y el ambiente tranquilo (Tabla 2).

En concordancia, la literatura ${ }^{(2,4,6,17,18)}$ menciona varios factores positivos para el cambio de turno, que incluyen, además de los resultados mencionados: la organización previa, la concentración mostrada, la puntualidad al principio y al final de procedimiento y el ambiente amplio. Todos estos son factores que contribuyen en la eficacia de este procedimiento, ya que es un tiempo esencial para asegurar la continuidad del cuidado de enfermería. En cuanto a la comparación entre las respuestas de los enfermeros y técnicos / auxiliares sobre estos factores, ambos mostraron una convergencia en la percepción de los factores positivos, lo que demuestra un nivel similar de satisfacción.

Tabla 2. Factores internos que favorecen el proceso de cambio de turno según reportado por los profesionales de enfermería de un hospital universitario. Sergipe, Brasil, 2012.

\begin{tabular}{|c|c|c|c|c|}
\hline Fortalezas & $\begin{array}{l}\text { Frecuencia } \\
\text { Enfermeros }\end{array}$ & $\%$ & $\begin{array}{l}\text { Frecuencia } \\
\text { Tec/Aux }\end{array}$ & $\%$ \\
\hline $\begin{array}{l}\text { Duración máxima de } 15 \text { a } 20 \text { minutos } \\
\text { para el cambio de turno }\end{array}$ & 21 & 11,93 & 69 & 11,29 \\
\hline Claridad de la información & 21 & 11,93 & 65 & 10,64 \\
\hline Objetividad de la información & 20 & 11,36 & 67 & 10,97 \\
\hline Entorno tranquilo & 20 & 11,36 & 62 & 10,15 \\
\hline $\begin{array}{l}\text { Agilidad en el intercambio de } \\
\text { información }\end{array}$ & 19 & 10,80 & 62 & 10,15 \\
\hline Cooperación del equipo & 18 & 10,23 & 69 & 11,29 \\
\hline Compromiso del personal & 18 & 10,23 & 68 & 11,13 \\
\hline El tipo de Líder & 14 & 7,95 & 47 & 7,69 \\
\hline Ambiente confortable & 10 & 5,68 & 29 & 4,75 \\
\hline El personal planifica el cambio de turno & 8 & 4,55 & 49 & 8,02 \\
\hline $\begin{array}{l}\text { Puntualidad para iniciar el cambio de } \\
\text { turno }\end{array}$ & 7 & 3,98 & 24 & 3,93 \\
\hline Total & 176 & 100 & 611 & 100 \\
\hline
\end{tabular}

Fonte: datos de la investigación

Sin embargo, los enfermeros indicaron como debilidades: funcionarios con prisa por salir, impuntualidad para iniciar el cambio de turno, atrasos del personal, conversaciones durante el cambio de turno y falta de planificación. Por otro lado, los técnicos / auxiliares también mencionaron como debilidades: retrasos de los compañeros, impuntualidad para iniciar el cambio de turno, personal con prisa para salir, conversaciones durante el cambio de turno y falta de planificación (Tabla 3). Con respecto a estas debilidades señaladas por ambas categorías, se observó similitud de las respuestas, que se refieren principalmente a la falta de planificación, protocolización y comprensión acerca de la importancia del proceso de cambio de turno, ya que los comportamientos observados en los profesionales demuestran una 
actitud inadecuada frente a la importancia que este procedimiento tiene para una atención de enfermería de calidad. Declaraciones que presentan una contradicción con respecto a los factores positivos mencionados por los propios profesionales, ya que mientras ellos informaron que existe compromiso y cooperación de los empleados para realizar el cambio de turno, también reclamaron de conductas no deseadas y que perjudican un cambio de turno efectivo, situación que es contraria a los principios de compromiso y cooperación antes mencionados.

Al respecto, investigaciones mencionan algunas dificultades durante el cambio de turno, las cuales comprometen este proceso, generando actitudes y comportamientos que dañan el cuidado, en detrimento de la calidad de la atención de enfermería $(3,4,6,18)$

Tabla 3. Factores internos que dificultan el proceso de cambio de turno, según reportan los profesionales de enfermería en un hospital universitario. Sergipe, Brasil, 2012

\begin{tabular}{|c|c|c|c|c|}
\hline Debilidades & $\begin{array}{l}\text { Frecuencia } \\
\text { Enfermeros }\end{array}$ & $\%$ & $\begin{array}{l}\text { Frecuencia } \\
\text { Tec/Aux }\end{array}$ & $\%$ \\
\hline $\begin{array}{l}\text { Los Profesionales de enfermería } \\
\text { con prisa para salir. }\end{array}$ & 19 & 16,96 & 49 & 13,10 \\
\hline Impuntualidad para iniciar. & 17 & 15,18 & 50 & 13,37 \\
\hline $\begin{array}{l}\text { Llegar tarde a la entrega de } \\
\text { turno }\end{array}$ & 16 & 14,29 & 59 & 15,78 \\
\hline $\begin{array}{l}\text { Conversaciones } \\
\text { entrega de turno }\end{array}$ & 13 & 11,61 & 46 & 12,30 \\
\hline Falta de planificación & 11 & 9,82 & 28 & 7,49 \\
\hline No valorizar el cambio de turno & 9 & 8,04 & 23 & 6,15 \\
\hline Falta de claridad en los registros & 7 & 6,25 & 27 & 3,21 \\
\hline Tipo de liderazgo & 7 & 6,25 & 12 & 3,21 \\
\hline $\begin{array}{l}\text { Ausencia de comunicación } \\
\text { directa entre los equipos de } \\
\text { trabajo }\end{array}$ & 5 & 4,46 & 26 & 6,95 \\
\hline Documentación insuficiente & 3 & 2,68 & 20 & 5,35 \\
\hline $\begin{array}{l}\text { Falta de claridad en la } \\
\text { comunicación verbal }\end{array}$ & 1 & 0,89 & 12 & 3,25 \\
\hline $\begin{array}{l}\text { Excesivo número de personas } \\
\text { en la estación de enfermería }\end{array}$ & 4 & 3,57 & 21 & 5,61 \\
\hline Total & 112 & 100 & 374 & 100 \\
\hline
\end{tabular}

Fuente: Datos de la investigación

En lo que respecta a las oportunidades como factor externo, los resultados mostraron que, según la percepción de los enfermeros, fueron identificados como externos positivos: la buena relación con otros profesionales de la salud y los descubrimientos científicos, como por ejemplo los nuevos tratamientos de salud. En este aspecto, los técnicos / auxiliares señalaron: la buena relación con otros profesionales de la salud, la administración del hospital y los nuevos modelos de gestión implementados en la institución (Tabla 4). 
Enfermería, como una práctica social y parte de una institución de salud, interactúa con otros profesionales favoreciendo el trabajo en equipo, interdisciplinario y humanizado para tratar de satisfacer todas las necesidades del paciente. En este sentido, investigaciones $(8,19,20,21)$ mencionan que los factores externos, como la búsqueda compartida de nuevos conocimientos, la interacción con diferentes profesionales, así como el compromiso de la gestión hospitalaria son esenciales para proporcionar un ambiente de continuo aprendizaje, así como una buena planificación de la asistencia.

Tabla 4. Factores externos que favorecen el proceso de cambio de turno según reportan los profesionales de enfermerías en un hospital universitario. Sergipe, Brasil, 2012.

\begin{tabular}{|c|c|c|c|c|}
\hline Oportunidades & $\begin{array}{l}\text { Frecuencia } \\
\text { Enfermeros }\end{array}$ & $\%$ & $\begin{array}{l}\text { Frecuencia } \\
\text { Tec/Aux }\end{array}$ & $\%$ \\
\hline $\begin{array}{l}\text { Las relaciones con } \\
\text { profesionales de la salud }\end{array}$ & 14 & 77,78 & 58 & 38,16 \\
\hline $\begin{array}{l}\text { Los descubrimientos científicos, } \\
\text { como nuevas formas de } \\
\text { tratamiento }\end{array}$ & 4 & 22,22 & 11 & 7,24 \\
\hline $\begin{array}{l}\text { Las nuevas tecnologías, como la } \\
\text { informatización }\end{array}$ & 0 & 0 & 0 & 0 \\
\hline $\begin{array}{l}\text { Nuevos modelos de gestión } \\
\text { implementados en la institución }\end{array}$ & 0 & 0 & 37 & 24,34 \\
\hline $\begin{array}{l}\text { La propia administración del } \\
\text { hospital }\end{array}$ & 0 & 0 & 46 & 30,26 \\
\hline Total & 18 & 100 & 152 & 100 \\
\hline
\end{tabular}

Fuente: Datos de la investigación

En cuanto a los factores externos negativos (amenazas), los enfermeros indicaron como amenazas: el exceso de unidades bajo la responsabilidad de un enfermero, la propia administración del hospital, las llamadas telefónicas durante el cambio de turno y las interrupciones médicas o de otros profesionales durante el cambio de turno. Los técnicos / auxiliares destacaron también las llamadas telefónicas durante el cambio de turno, el exceso de unidades bajo la responsabilidad de un mismo enfermero, la propia administración del hospital y las interrupciones médicas 0 de otros profesionales durante el cambio de turno (Tabla 5).

El procedimiento de cambio de turno puede ser perjudicado debido a la sobrecarga en la supervisión tanto de pacientes como de profesionales. En este contexto, las dificultades encontradas en este estudio alertan sobre problemas asistenciales y administrativos existentes en el contexto estudiado, tales como la falta de información acerca del turno anterior, lo que interfiere sin duda con la eficacia de la entrega de turno ${ }^{(3,4,6)}$. 
Tabla 5. Factores externos que obstaculizan el proceso de cambio de turno según los profesionales de enfermería en un hospital universitario. Sergipe, Brasil, 2012.

\begin{tabular}{lccccc}
\hline Amenazas & $\begin{array}{l}\text { Frecuencia } \\
\text { Enfermeros }\end{array}$ & $\%$ & $\begin{array}{l}\text { Frecuencia } \\
\text { Tec/Aux }\end{array}$ & $\%$ \\
\hline $\begin{array}{l}\text { Exceso de unidades bajo } \\
\text { responsabilidad de un enfermero } \\
\text { La propia administración del hospital }\end{array}$ & 14 & 25 & 45 & 19,91 \\
$\begin{array}{l}\text { Llamadas telefónicas durante el cambio } \\
\text { de turno }\end{array}$ & 12 & 23,21 & 39 & 17,26 \\
$\begin{array}{l}\text { Interrupciones médicas o de otros } \\
\text { profesionales durante el cambio de } \\
\text { turno }\end{array}$ & 9 & 16,07 & 33 & 14,60 \\
$\begin{array}{l}\text { Unidades con excesivo número de } \\
\text { pacientes }\end{array}$ & 4 & 7,14 & 27 & 11,95 \\
$\begin{array}{l}\text { Flujo de personal que no pertenece a la } \\
\text { unidad }\end{array}$ & 2 & 3,57 & 14 & 6,19 \\
$\begin{array}{l}\text { Relaciones con otros profesionales de } \\
\text { la salud }\end{array}$ & 1 & 1,79 & 11 & 4,87 \\
$\begin{array}{l}\text { Nuevos modelos de gestión } \\
\text { implantados en la institución }\end{array}$ & 1 & 1,79 & 8 & 3,54 \\
\hline \begin{tabular}{l} 
Total \\
\hline
\end{tabular} & 56 & 100 & 126 & 100 \\
\hline
\end{tabular}

Fuente: Datos de la investigación

Al respecto se observaron similitudes en las respuestas de los enfermeros y los técnicos / auxiliares en cuanto a la administración del hospital, lo cual mostró insatisfacción de los profesionales, debido especialmente a la falta de apoyo de la administración para conseguir un efectivo cambio de turno. Se insiste en que la prestación de mejores servicios de salud sólo se logra con la colaboración de la administración hospitalaria, así como por medio de la implementación de nuevos modelos de gestión en la institución ${ }^{(8,20)}$.

Tras el análisis y el estudio sistemático de los factores internos y externos fue posible construir la matriz FODA (Tabla 1), donde se muestran alternativas estratégicas. Éstas se basaron en la utilización de las fortalezas y en la corrección de las debilidades, así como aprovechar las oportunidades y mitigar las amenazas, favoreciendo así el alcance de la misión, visión y objetivos institucionales. Es de destacar que la planificación estratégica tiene como objetivo proponer estrategias que permitan el alcance de los objetivos, que proporcionen un mejor uso de los recursos, así como permite preparar a la institución para cambios probables ${ }^{(10)}$.

Frente a este contexto, las estrategias que se presentaron para el procedimiento de cambio de turno en el hospital universitario estudiado, mostraron el cotidiano de trabajo de los profesionales de enfermería, lo que permite repensar en nuevas perspectivas, en busca de posibles cambios en la gestión. Las estrategias fueron denominadas de Estrategias FO (utiliza las fortalezas para aprovechar las oportunidades), cuyo objetivo es: crear un entorno más propicio para el cambio de turno, mejorar los servicios prestados por el personal de enfermería, mejorar el comportamiento del equipo durante el cambio de turno, incrementar los conocimientos pertinentes para permitir un eficaz cambio de turno; Estrategias FA (utiliza las 
fortalezas para evitar las amenazas), cuyo objetivo es: crear una herramienta que facilite aún más la claridad de la información, la velocidad y la objetividad durante el intercambio de información, facilitar el cambio de turno para los enfermeros reduciendo así la carga de trabajo, evitar interrupciones durante el cambio de turno, y sensibilizar a la administración del hospital acerca de la importancia del cambio de turno.

Por otro lado, las estrategias de DO (toma ventaja de las oportunidades, superando las debilidades) su objetivo es: reducir las conductas inapropiadas en el informe de cambio de turno; estandarizar el tiempo para el cambio de turno, y finalmente las estrategias DA (evita las amenazas reduciendo al mínimo las debilidades), tales como: tratar de reducir la falta de planificación para el cambio de turno.

\section{Cuadro 1. Matriz FODA relativo al procedimiento de cambio de turno en el hospital universitario de Sergipe. Brasil, 2012.}

\begin{tabular}{|c|c|c|}
\hline & $\begin{array}{l}\text { OPORTUNIDADES } \\
\text { Las buenas relaciones con otros } \\
\text { profesionales. } \\
\text { Los descubrimientos científicos. } \\
\text { La propia administración del } \\
\text { hospital. } \\
\text { Nuevos modelos de gestión. }\end{array}$ & $\begin{array}{l}\text { AMENAZAS } \\
\text { El exceso de unidad bajo la } \\
\text { responsabilidad de un solo } \\
\text { enfermero. } \\
\text { La propia administración del } \\
\text { hospital. } \\
\text { Las llamadas telefónicas } \\
\text { durante el cambio de turno. } \\
\text { Las interrupciones médicas y } \\
\text { de otros profesionales }\end{array}$ \\
\hline $\begin{array}{l}\text { FORTALEZAS } \\
\text { Claridad de la } \\
\text { información. } \\
\text { Duración de máx. } \\
15 \text { a } 20 \text { minutos } \\
\text { para el cambio de } \\
\text { turno. } \\
\text { La objetividad de la } \\
\text { información. } \\
\text { Entorno tranquilo. } \\
\text { Agilidad en el } \\
\text { intercambio de } \\
\text { información. } \\
\text { Cooperación } \\
\text { compromiso entre el } \\
\text { equipo profesional. }\end{array}$ & $\begin{array}{l}\text { ESTRATEGIAS FO } \\
\text { Establecimiento de un local } \\
\text { específico para las reuniones } \\
\text { del cambio de turno. } \\
\text { Creación de un grupo de } \\
\text { estudio para identificar los } \\
\text { principales diagnósticos y } \\
\text { prescripciones de enfermería de } \\
\text { la unidad, a fin de enriquecer el } \\
\text { intercambio de información } \\
\text { durante el cambio de turno. } \\
\text { El enfermero, de acuerdo con la } \\
\text { administración del hospital, } \\
\text { realizará reuniones periódicas } \\
\text { de formación continua con el } \\
\text { personal de enfermería, con una } \\
\text { duración de hasta } 20 \text { minutos } \\
\text { sobre temas de interés y } \\
\text { resaltantes para el } \\
\text { procedimiento de cambio de } \\
\text { turno. }\end{array}$ & $\begin{array}{l}\text { ESTRATEGIAS FA } \\
\text { Elaborar un impreso para } \\
\text { anotación de informaciones } \\
\text { relevantes para ser } \\
\text { transmitidas durante el } \\
\text { cambio de turno. } \\
\text { Utilizar una tarjeta con la } \\
\text { siguiente información: "Por } \\
\text { favor, no interrumpir, hora del } \\
\text { cambio de turno". } \\
\text { La enfermera responsable de } \\
\text { la unidad enviará } \\
\text { mensualmente a la dirección } \\
\text { del hospital, un informe sobre } \\
\text { el desempeño de su equipo } \\
\text { durante el cambio de turno, } \\
\text { así como el resultado de los } \\
\text { indicadores de salud en la } \\
\text { misma unidad }\end{array}$ \\
\hline
\end{tabular}




\begin{tabular}{|c|c|c|}
\hline $\begin{array}{l}\text { DEBILIDADES } \\
\text { Profesionales } \\
\text { apresurados para } \\
\text { salir. } \\
\text { Impuntualidad para } \\
\text { iniciar el cambio de } \\
\text { turno } \\
\text { Colegas llegan } \\
\text { atrasados. } \\
\text { Conversaciones } \\
\text { durante el cambio } \\
\text { de turno. } \\
\text { La falta } \\
\text { planificación de }\end{array}$ & $\begin{array}{l}\text { ESTRATEGIAS DO } \\
\text { Establecer un horario fijo para el } \\
\text { inicio y el final del cambio de } \\
\text { turno. } \\
\text { Todos los empleados deben } \\
\text { completar el formulario } \\
\text { correspondiente, aclarando a } \\
\text { los responsables del cambio de } \\
\text { turno, si necesario, sobre la } \\
\text { información contenida en el } \\
\text { respectivo formulario. } \\
\text { El enfermero responsable de la } \\
\text { unidad deberá avisar a su } \\
\text { equipo de trabajo para que se } \\
\text { concentren únicamente en } \\
\text { cuestiones relativas a la entrega } \\
\text { de turno. }\end{array}$ & $\begin{array}{l}\text { ESTRATEGIAS DA } \\
\text { El enfermero debe } \\
\text { determinar qué empleados } \\
\text { serán responsables por el } \\
\text { cambio de turno siguiente. }\end{array}$ \\
\hline
\end{tabular}

Fuente: elaboración propia

\section{CONCLUSIONES}

El análisis de datos permitió construir consideraciones importantes para comprensión de la gestión del trabajo de enfermería, especialmente el referido al cambio de turno, identificando así retos y aspectos críticos.

Frente a esta situación, cuando analizados los factores internos y externos, se encontraron estrategias para la acción coherente con los resultados de la construcción de la matriz FODA, lo que facilitará el proceso de cambio de turnos en la institución estudiada.

Vale la pena señalar que, para lograr una atención de calidad se requiere la colaboración directa de la administración del hospital, especialmente en el procedimiento de cambio de turno. La transmisión de información durante el cambio de turno debe ser objetiva, aunque concisa, para garantizar la comprensión y la fluidez de la información.

El presente estudio permitió indicar importantes contribuciones para una gestión eficaz de entrega de turno, un procedimiento que a menudo no cumple su debido rol, causando perjuicios a los clientes y a la institución. El análisis de la planificación estratégica en el presente estudio trató de romper con los paradigmas existentes en el personal de enfermería para lograr y concretizar la calidad de atención deseada.

\section{REFERENCIAS BIBLIOGRÁFICAS}

1. Santos M C, Bernardes A. Comunicação da equipe de enfermagem e a relação com a gerência nas instituições de saúde. Rev. Gaucha de Enferm. 2010 jun; 31 (2):359-66.

2. Portal KM, Magalhães AMM. Passagem de plantão: um recurso estratégico para a continuidade do cuidado em enfermagem. Rev Gaúcha de Enferm. 2008 jun; 29 (2): 246-56.

3. Siqueira ILCP, Kurcgant P. Passagem de plantão: falando de paradigmas e estratégias. Acta Paul Enferm. 2005, 18(4), 446-51. 
4. Zoehler KG, Lima MADS. Opinião dos auxiliares de enfermagem sobre a passagem de plantão. Rev. Gaúcha Enferm. 2000, 21(2): 110-24

5. Timby BK. Conceitos e habilidades fundamentais no atendimento de enfermagem. 8.ed. Rio Grande do Sul: Ed. Artmed S.A; 2005.

6. Andrade JS, Vieira MJ, Lima D M, Santana MA. A comunicação entre enfermeiros na passagem de plantão. Acta Paul Enferm. 2004 jul-set;17(3): 311-5.

7. Kurcgant $P$ et al. Gerenciamento em Enfermagem. Rio de Janeiro: Ed. Guanabara Koogan, 2011.

8. Ciampone MHT, Melleiro M. O planejamento e o processo decisório como instrumentos do processo de trabalho gerencial. In: Kurcgant P. Gerenciamento em Enfermagem. Rio de Janeiro: Guanabara Koogan; 2011. p.35-50.

9. Chiavenato, I. Administração: teoria, processo e prática. 3.ed. São Paulo: MakronBooks, 2004.

10. Terence ACF. Planejamento estratégico como uma ferramenta de competitividade na pequena empresa: desenvolvimento e avaliação de um roteiro prático para o processo de elaboração do planejamento [dissertação]. São Paulo: Escola de Engenharia de São Carlos; 2002.

11. Carvalho et al. Estratégias para organização da assistência farmacêutica da farmácia especial. In:Peixe, B C S et al. Gestão de políticas públicas no Paraná: Coletânea de estudos. Curitiba: Ed. Progressiva; 2008.

12. Stoner J A F, Freeman R E. Administração. 5ae ed. Rio de Janeiro (RJ): LTC, 1999.

13. Mintzberg $\mathrm{H}$. The fall and rise of strategic planning. Boston: Harvard Business Review. 1994. jan-feb; 72(1): 107-14.

14. Aquino LAM, Teodoro W R. Análise do processo de passagem de plantão em uma unidade de internação pediátrica. Rev. Min. Enferm. 2010 jul-set; 14(3): 316-26.

15. Matheus MCC, Colvero LA, Igue CE, Dias DC. Passagem de plantão: um estudo da comunicação verbal e não verbal. Acta Paul Enf.1998; 11(2): 77-82.

16. Penaforte MHO, Martins MMFPS. A visibilidade do autocuidado relativo à higiene na passagem de plantão dos enfermeiros. Rev. Latino-Am. Enfermagem [Internet]. jan-fev 2011[acesso em: 01 marc. 2011];19(1):[09 telas]. Disponível em: www.eerp.usp.br/rlae

17. Krutinsky DC, Cornacchia H, Coutinho RMC, Leitão IC, Souza JC, Ananias JC. O significado da passagem de plantão por trabalhadores de enfermagem. Ver. Inst.Ciênc. Saúde. 2007; 25(2): 105-11.

18. Pirolo SM, Chaves EC. A equipe de enfermagem e o mito do trabalho em grupo. Rev. Esc. Enferm.2002; 36(4): 351-7.

19. Vilas Boas MAV. Passagem de plantão em um hospital psiquiátrico [dissertação]. São Paulo: Escola de Enfermagem de Ribeirão Preto; 2004.

20. Bernardino E, Felli VEA, Peres AM. Competências gerais para o gerenciamento em enfermagem de hospitais. Cogitare Enferm. 2010 abr-jun; 15(2): 349-53.

21. Peres AM, Ciampone MHT. Gerências e competências gerais do enfermeiro. Texto e Contexto Enferm. 2006 jul-set; 15(3): 492-9.

22. Lip C. Administración en Salud. Universidad Nacional de San Agustin. Facultad de Enfermeria. Programa de Maestria en Enfermería. 1998; 63.

ISSN 1695-6141

๑ COPYRIGHT Servicio de Publicaciones - Universidad de Murcia 